\title{
La construcción de un indicador de trayectoria ocupacional
}

\author{
The construction of an occupational trajectory indicator
}

\author{
Ruth Sautu \\ Instituto de Investigaciones Gino Germani, \\ Universidad de Buenos Aires (IIGG-UBA), Argentina \\ Joaquín Carrascosa \\ Instituto de Investigaciones Gino Germani, \\ Universidad de Buenos Aires (IIGG-UBA), Argentina \\ Diego Paredes \\ Instituto de Investigaciones Gino Germani, \\ Universidad de Buenos Aires (IIGG-UBA), Argentina
}

Cita sugerida: Sautu, R., Carrascosa, J. y Paredes, D. (2020). La construcción de un indicador de trayectoria ocupacional. Revista Latinoamericana de Metodología de las Ciencias Sociales, 10(2), e075. https://doi.org/10.24215/18537863e075

Recepción: 25 Mayo 2020

Aprobación: 15 Octubre 2020

Publicación: 01 Diciembre 2020

\begin{abstract}
Resumen: El objetivo de este artículo es describir un procedimiento para la construcción de uno de los indicadores de la variable trayectoria ocupacional, en el cual se comparan la ocupación desempeñada a los 25 años y la actual (o última en el caso de retirados/jubilados). El estudio en el cual se usará el indicador tiene como objetivo general analizar los procesos de reproducción y cambio ocupacional, en el marco de las clases sociales. Los datos provienen de una encuesta aleatoria multietápica realizada en 2015-2016 entre 1065 miembros de hogares residentes en el Área Metropolitana de Buenos Aires $(\mathrm{AMBA})$, de la cual se ha seleccionado un subuniverso de mujeres y varones de entre los 35 y 50 años de edad. En el procesamiento de los datos para la construcción del indicador de corte transversal se utilizó el paquete estadístico Stata.
\end{abstract}

Palabras clave: Indicador nominal de corte transversal, Trayectoria ocupacional, Paquete estadístico stata.

Abstract: The aim of this paper is to describe the procedure for the construction of one of the indicators of a variable of occupational trajectory in which we compare the occupation at twenty-five years of age and their current occupation (or the last occupation in the case of retirees). The study in which the indicator will be used has as a general aim to analyze the processes of reproduction and occupational change, in the framework of social classes. The data comes from a random multi-stage survey done in 2015-2016 between 1065 members of households from the Metropolitan Area of Buenos Aires (AMBA), from which we have selected a sub-set of men and women between 35 and 50 years old. For the processing of the data for the construction of the transversal indicator we used the statistical software Stata.

Keywords: Transversal nominal indicator, Occupational trajectory, Stata statistical software.

\section{INTRODUCCIÓN}

El objetivo de este artículo es describir un procedimiento para la construcción de uno de los indicadores de la variable trayectoria ocupacional, en el cual se comparan la ocupación desempeñada a los 25 años y la actual (o última en el caso de retirados/jubilados). Este indicador se ubica en las etapas iniciales de una investigación por encuesta cuando se procede a la selección y construcción de los indicadores de las variables. Su propósito es comparar desempeño de ocupaciones; su nivel de medición es nominal. 
El estudio en el cual se usará el indicador tiene como objetivo general analizar los procesos de reproducción y cambio ocupacional, en el marco de las clases sociales. Los datos provienen de una encuesta aleatoria multietápica realizada en 2015-2016 entre 1065 miembros de hogares residentes en el Área Metropolitana de Buenos Aires (AMBA). ${ }^{1}$

En su perspectiva teórica la investigación, en la cual será usado el presente indicador, postula que las trayectorias ocupacionales forman parte de los procesos de reproducción y cambio de las clases sociales en los cuales el curso de vida de las personas y su capacidad de agencia se insertan en las condiciones, oportunidades y limitaciones de la estructura económica y los mercados laborales. Al respecto se tiene en cuenta que los mercados de trabajo históricamente constituidos funcionan sobre la base de competencias y discriminan según rasgos físicos, sociológicos y culturales. Aunque en buena medida ellos pertenecen al ámbito de la economía, en su accionar están entretejidos con la sociedad, su cultura y los procesos de exclusión y discriminación (Sautu, 2011 y 2016).

El presente artículo se inicia describiendo la perspectiva teórica en la cual se ubica la definición conceptual de trayectoria ocupacional. Subsecuentemente se sintetizan algunos estudios sobre trayectorias ocupacionales y su medición empírica sobre los cuales se apoyó nuestro estudio para la selección de los criterios y el procedimiento usado en la construcción del indicador. En la conclusión se reflexiona sobre la utilidad y limitaciones del indicador.

\section{EL CONCEPTO TRAYECTORIA OCUPACIONAL}

El concepto trayectoria ocupacional forma parte de la perspectiva teórico-metodológica de los cursos de vida. En esta perspectiva cuantitativa se ubica la investigación para la cual hemos construido el indicador de trayectorias. Dos supuestos están presentes en el diseño de ese estudio y por lo tanto en la construcción de sus indicadores: la inserción de la trayectoria en el mercado de trabajo y su conceptualización como parte de las clases sociales.

Primero, las investigaciones sobre el curso de vida y la reconstrucción de las trayectorias de vida, en perspectiva longitudinal, se asientan en un contexto socioestructural histórico; uno de sus propósitos es la articulación entre ese contexto y el microcosmos de las decisiones y orientaciones individuales y la acción social. Por lo tanto, las trayectorias ocupacionales, residenciales y educativas, los sucesos de la vida familiar y los lazos sociales se entrelazan con las circunstancias de los cambios socioestructurales, políticos y poblacionales en los cuales ellas ocurren. Así, la investigación de cursos de vida (life course research) consiste fundamentalmente en establecer los patrones de comportamiento por edad que se encuentran insertos en las instituciones y la historia; son caminos sociales en el tiempo y espacio histórico (Elder, Johnson \& Crosnoe, 2006, p. 4).

El contexto estructural histórico establece condiciones de oportunidades y limitaciones en los mercados laborales.

Los patrones que siguen las trayectorias laborales reflejan no sólo los distintos arreglos institucionales particulares de cada sociedad, sino también la mayor heterogeneidad interna de cada mercado de trabajo (...) Por ello, el análisis de las trayectorias ocupacionales en las sociedades en desarrollo puede proporcionar conocimiento en torno a los determinantes institucionales de los cursos de vida ocupacionales. En el contexto específico de México, el estudio de las trayectorias ocupacionales es también relevante porque permite rastrear algunos de los efectos del cambio estructural sobre los cursos de vida (Solís y Billari, 2003, p. 560).

El segundo supuesto teórico es que los cursos de vida, y por ende las trayectorias, ocurren en el marco de las clases sociales; de las oportunidades y limitaciones que establece la pertenencia a clase en los mercados laborales, los cuales diferencian en términos de competencias ocupacionales y de rasgos personales. Las personas crean su propia vida a través de elecciones y decisiones; la clase social, el género y la etnia establecen 
los límites de las probabilidades de accionar de la agencia humana. Incluir las clases sociales en el enfoque de los cursos de vida permite develar cómo se entretejen longitudinalmente los aspectos micro, meso y macrosociales que subyacen a la conformación de la estructura de clase, de la movilidad o la herencia de clase. El enfoque biográfico, en su vertiente cuantitativa o cualitativa, permite estudiar los recursos materiales y esquemas de acción reconstruidos desde el actor-sujeto; asimismo es posible indagar, desde su perspectiva, las relaciones sociales y legados culturales que se trasmiten al interior de la trama familiar en relación con las transformaciones del contexto sociohistórico.

En síntesis, en la perspectiva teórico-metodológica de cursos de vida, el concepto trayectoria ocupacional puede definirse de la siguiente manera: primero, constituye un patrón que caracteriza comportamientos de inserción ocupacional en los mercados de trabajo a lo largo del tiempo; el inicio, así como la culminación constituyen hitos claves en dicho patrón. Segundo, el lugar de residencia, la familia, la escuela a la cual se asiste, el lenguaje que se habla, en síntesis, las competencias ocupacionales están asociadas a la clase social. Y tercero, los mercados de trabajo están insertos en los procesos histórico-económicos, políticos y sociales.

Esa definición requiere que en la construcción del indicador se tenga en cuenta: primero, la categorización de ocupaciones en términos de competencias estratificadas; es decir de diferencias jerárquicas establecidas por los recursos materiales e inmateriales que requieren su desempeño. Y segundo, incorporar en la medición el tramo del tiempo con el fin de establecer reproducción o cambio, y la dirección ascendente o descendente del mismo.

La definición conceptual de la variable trayectoria ocupacional como la construcción del indicador se ha nutrido en los antecedentes de estudios sobre el tema. La multiplicidad de enfoques, cualitativos y cuantitativos, se encuentra en Muñiz Terra (2012), quien sistematiza una amplia bibliografía sobre los enfoques teóricos y perspectivas metodológicas utilizadas en el estudio de las carreras y trayectorias laborales, destacando asimismo sus potencialidades y limitaciones. Desde la perspectiva cualitativa define trayectoria como un entramado de experiencias laborales, lo que permite reconstruir el encadenamiento de sucesos en la vida de las personas. En esta línea de trabajo cualitativa ha analizado investigaciones que tratan el desempleo, la relación con el sistema educativo, el género, la movilidad sociolaboral y las condiciones de vida, la identidad y cultura obrera, y las estrategias de trabajadores informales. En la perspectiva cuantitativa se han estudiado las trayectorias como las secuencias de categorías ocupacionales en un periodo determinado; lo que permite captar la variabilidad ocupacional de distintos sectores poblacionales a la luz de los cambios que suceden en el mercado laboral. Como ejemplo menciona los estudios sobre trabajadores despedidos, el impacto de las privatizaciones y asalariados del sector privado. $^{2}$

\section{CRITERIOS PARA LA CONSTRUCCIÓN DEL INDICADOR}

Empíricamente la trayectoria está constituida por todas las ocupaciones desempeñadas a lo largo de la vida económicamente activa, las cuales han sido reconstruidas retrospectivamente por la persona entrevistada. Se trata por lo tanto de una selección procesada en el presente a través de las interpretaciones subjetivas de experiencias pasadas. Además de la información sobre las ocupaciones, las encuestas incluyen la edad, fecha y el lugar donde fueron desempeñadas.

El periodo y la cohorte de nacimiento ubican históricamente la trayectoria; los tramos de edad a su vez especifican las posibilidades y limitaciones en el mercado de trabajo. Dos momentos son considerados los más importantes: el inicio de la vida laboral y la etapa adulta joven. En la práctica de los estudios en demografía y educación se usan los 17-18 años como edad de finalización de la escuela secundaria, y los 24-25 años como edad promedio de terminación de los estudios terciarios y universitarios. A su vez, la investigación sobre participación económica, empleo y desempleo utilizan la agrupación quinquenal de edades, comenzando por 15-19 años, y siguiendo con 20-24 años de edad (así cada cinco años). 
Durante el primer tramo de edad los estudios analizan el papel que juegan la educación y la familia en el acceso al mercado laboral y a futuro en las probabilidades de movilidad ocupacional. Algunos análisis de la movilidad inter o intrageneracional tienen en cuenta el papel de la educación y también las habilidades cognitivas y conocimientos adquiridos a una edad temprana (Karlson \& Birkelund, 2019).

El tramo de la vida adulta joven, a partir de los 25 años, se caracteriza porque se ha completado la etapa de educación formal y/o de adquisición de competencias en el desempeño laboral, sean estas las de profesionales universitarios o las que se requieren para la práctica en el desempeño laboral (por ejemplo, en albañilería). En particular para miembros de la clase popular, discontinuidad e inestabilidad caracterizan algunas trayectorias juveniles (Busso, Bidart, Longo y Pérez, 2011; Sautu, 2020), proceso este que está asociado al crecimiento de actividades terciarias y del sector informal. Como señalan Jacinto y Chitarroni (2010) en su investigación de trayectorias laborales juveniles, la transición hacia el trabajo de jóvenes, de entre 19 y 29 años, "se caracteriza por la diversidad, la inestabilidad y la precariedad de las experiencias laborales, y está influida no solo por la inestabilidad estructural del mercado de empleo sino también (...) por ser joven” (p. 3).La transformación y crisis de las economías capitalistas avanzadas, según algunos estudios, está generando también, entre otras consecuencias, discontinuidades e inestabilidad en trayectorias laborales juveniles (Struffolino, 2019).

La etapa adulta joven comprende las edades entre los 24/25 años de edad hasta alrededor de los 50 años. Las curvas de participación económica por edad justamente muestran que las tasas por edad son las más altas durante las edades centrales; y aunque los niveles masculinos son superiores a los femeninos, esa regularidad se observa en ambos sexos. Más aun, mientras las distribuciones de ingresos por edad indican que son más altos en esos tramos centrales de edad, cuando se computan las tasas de desempleo estas son más bajas que en ambos extremos, los tramos de edad de los jóvenes y los adultos de mayor edad (como contexto social y económico, ver Lindenboim, 2001; Lindenboim y Salvia, 2016; Danani y Lindenboim, 2003).

En el contexto histórico-económico de los mercados laborales y en la perspectiva de las clases sociales que establecen oportunidades y limitaciones diferenciales, el tramo de edad adulta joven constituye una etapa de la trayectoria ocupacional durante la cual asumimos que con mayor frecuencia tienen lugar los procesos de cambio ocupacional. Tenemos en cuenta que, mientras en el tramo de edad de inicio de la vida económicamente activa la influencia del origen de clase y de los lazos y relaciones familiares es fundamental, durante la vida adulta joven la educación formal y la adquisición de conocimientos y competencias devienen cruciales en el mercado de trabajo para explicar los procesos de reproducción, ascenso o descenso ocupacional.

Existen dos maneras básicas de sintetizar el análisis de trayectorias, el de corte transversal y el longitudinal. El primero permite describir los cambios netos ocurridos entre ambas mediciones; su propósito es principalmente comparativo entre conjuntos poblacionales (clases sociales, género, territorios, etc.). El enfoque longitudinal está basado en la idea de que los individuos no pueden ser simplemente clasificados de acuerdo a sus características en un solo punto en el tiempo. Así, el tiempo y el curso de vida deben ser considerados significativos cuando se describen secuencias de datos (Rohwer y Trappe, 1999, en Billari, 2001).

El indicador aquí descripto pertenece a la categoría de corte transversal, ya que se utilizará para comparar diferentes conjuntos sociales. Dos preguntas subyacen a estos análisis: ¿cuál es el peso de la reproducción o del cambio ocupacional? ¿Existen diferencias entre distintos periodos históricos? Mientras en la primera se comparan por ejemplo clases sociales, la segunda nos permite ver un aspecto de los procesos de desarrollo económico-político de distintos periodos históricos, los cuales se plasman en los mercados laborales.

\section{LA CONSTRUCCIÓN DE LOS DATOS}

La trayectoria es una urdimbre longitudinal cuyos hilos representan diversos elementos de la historia personal; entre ellos nos interesan los siguientes. Primero, identificar a las personas. Segundo, conocer el tipo de ocupaciones desempeñadas, ya que a partir de ellas podremos establecer en qué consiste la reproducción o 
el cambio y el sentido de este último. Y tercero, el periodo histórico en el cual ellas son desempeñadas, porque esto nos permitirá acotar los procesos económicos que constituyen su contexto histórico. Esta selección pragmática nos sirve para estandarizar algunos rasgos claves de las trayectorias objeto del presente análisis. El número de elementos seleccionados está condicionado por el tamaño de la muestra. En la construcción del indicador de la variable trayectoria tendremos en cuenta esos tres elementos. Más adelante, en el estudio para el cual este indicador es construido, incorporaremos al análisis otras variables.

La base de datos con la que contamos, importante para muchos análisis, limita el número de variables y de categorías que es posible incorporar válidamente en un tabulado. Nuestra muestra de 1065 casos incluye personas de ambos sexos de 25 a 65 años de edad cuya vida laboral, muy probablemente, como conjunto, ha transcurrido entre 1966 y 2015, momento de la encuesta. En este largo periodo tuvieron lugar modelos distintos de desarrollo económico, razón por la cual decidimos acotarlo al periodo que se da entre los años 1990 y 2015, durante el cual, como conjunto, podemos asumir que transcurrió la vida laboral de los encuestados que en la última fecha tenían entre 35 y 50 años. El presente análisis, por lo tanto, ha seleccionado los encuestados de ambos sexos con edades entre 35 y 50 años de edad. Los mismos argumentos apoyan la decisión de tomar como hitos claves de la trayectoria las ocupaciones desempeñadas a los 25 años y las actuales (o última en el caso de retirados/jubilados).

La decisión de considerar la ocupación desempeñada a los 25 años y compararla con la actual se apoya además en la evidencia recogida en el estudio biográfico de trayectorias (Sautu, 2020, en evaluación). En este trabajo reentrevistamos a 23 personas de ambos sexos que habían respondido a la encuesta aleatoria; entre estos entrevistados seleccionamos 10 varones adultos jóvenes de entre 34 y 45 años de edad, y a partir de sus relatos pormenorizados reconstruimos sus trayectorias ocupacionales desde sus primeros trabajos. Aunque no es posible generalizar al universo, sirve de indicio saber: primero, la clase social de origen y la educación recibida, sobre todo el abandono de la escuela secundaria, daban cuenta de la edad de inicio de las experiencias laborales; segundo, aunque existen casos de personas con un patrón permanente de inestabilidad laboral, existe una tendencia a la mayor permanencia en los tipos de competencias desempeñadas (no así necesariamente en los puestos de trabajo) alrededor de los 23/25 años de edad; y tercero, las trayectorias están asociadas al origen de clase y el nivel de educación recibida. Todos estos datos son consistentes con los análisis de movilidad inter e intrageneracional realizados en nuestro país.

La decisión de acotar la franja etaria del análisis (35 a 50 años de edad, de personas de ambos sexos) se justifica porque deseamos subsecuentemente saber del menú de tipos inferidos de los datos cuáles son típicos femeninos y en cuáles predominan los varones durante el tramo de edades adultas-jóvenes. Esto posiblemente constituya un indicio de la discriminación por género de los mercados laborales, ya que un criterio central para la construcción del indicador de trayectoria ocupacional es el tipo de ocupación desempeñada.

La base de datos con la que trabajamos ha codificado cada ocupación en la trayectoria con el Código Internacional Uniforme de Ocupaciones de la OIT (CIUO-08). Las categorías de este código han sido reagrupadas con el fin de tener un número menor de categorías en una franja más acotada de competencias y jerarquías. La Tabla 1 del Anexo despliega cómo han sido agrupadas en cinco conjuntos las ocupaciones del CIUO, a cuatro dígitos (la mayor desagregación). Cada uno de esos cinco conjuntos contiene ocupaciones que han sido incluidas teniendo en cuenta cómo fueron categorizadas en un estudio sobre la estructura de clase: ocupaciones medias altas, medias-medias, media-baja, trabajadores-obreros calificados/especializados, y trabajadores-peones no calificados. Estos cinco subconjuntos coinciden aproximadamente con las clases sociales que constituyen la estructura de clase del AMBA construida con los datos de la misma encuesta aleatoria (Sautu, en Sautu, Boniolo, Dalle y Elbert, 2020).

Entendemos que es válido usar las posiciones de la estructura de clase para construir las categorías de la Tabla 1, porque en la perspectiva teórica de este estudio señalamos que la estructura económica y la estructura de clase se corresponden ontológicamente, mientras que la estructura ocupacional que es una consecuencia de la primera es utilizada como indicador de la segunda. Las relaciones de clase social, que 
son relaciones de poder, se despliegan, se corporizan, en y durante el desempeño ocupacional dentro de las organizaciones económicas, sean grandes corporaciones, organismos del Estado, o unidades unipersonales o familiares (Sautu, 2011 y 2016).

La base para la construcción del indicador es un tabulado de los datos de varones y mujeres de 35 a 50 años de edad. En dicho tabulado (Tabla 2 del Anexo), identificados y agrupados según el código de la Tabla 1, se han cruzado los datos de la ocupación a los 25 años por la ocupación actual (en 2015/2016).

El procedimiento para construir tipos de asociación entre las categorías de las ocupaciones actual y a los 25 años consistió en: primero, en la Tabla 2, identificar y tratar separadamente cada conjunto de la ocupación actual; la lectura horizontal de la Tabla 2 nos indica, de los que actualmente desempeñan una ocupación, qué ocupaciones desempeñaban a los 25 años. Segundo, identificar el cuadrante de la Tabla 2 en el cual coinciden las ocupaciones a los 25 años con la actual. Tercero, sumar las frecuencias dentro de dicho cuadrante. Cuarto, identificar para cada ocupación actual los cuadrantes ubicados a su derecha y a su izquierda. Quinto, la suma de las frecuencias de los cuadrantes ubicados a la izquierda muestra los casos en los cuales se produjo descenso ocupacional. Sexto, la suma de las frecuencias ubicadas a la derecha muestra los casos de ascenso ocupacional.

El Cuadro 2, incluido más adelante, muestra las combinaciones obtenidas a partir del análisis anterior y sus frecuencias (Tabla 2 del Anexo). Por ejemplo, Directores y Profesionales Universitarios, sobre el total de esa ocupación de 78 casos, a los 25 años ya se desempeñaban como tales 36; habían ascendido desde las ocupaciones Media-medias 28 casos, y desde obreros y peones 14 casos. Otro ejemplo del Cuadro 2 son los obreros calificados: sobre un total de 137 casos, a los 25 años ya eran obreros calificados 100 casos, descendía desde las clases medias 25 y ascendían desde peones 12 casos.

\section{EL PROCESAMIENTO DE DATOS Y LA CONSTRUCCIÓN DE UN INDICADOR DE TRAYECTORIA OCUPACIONAL}

En esta sección describiremos el uso de Stata para el procesamiento de los datos de trayectorias laborales. Stata es un software de procesamiento y análisis de datos cuantitativos que permite realizar rápida y eficientemente todos los procedimientos requeridos para la creación de variables y el análisis de cuadros, entre muchas otras cosas. Se describirá el filtrado de la base de datos para que coincida con la selección del universo de casos a analizar, la creación de la variable de ocupación a los 25 años de edad, la reagrupación de la variable ocupación desde el CIUO-08 hasta sistemas de menor número de categorías y la construcción de un indicador de corte transversal de las trayectorias ocupacionales.

El primer paso fue "filtrar" según la selección de casos realizada (personas de ambos sexos entre 35 y 50 años de edad). Esto puede realizarse en Stata de varias maneras, pero en este caso utilizamos el comando "drop", que nos permitió remover los casos que no queremos tener en cuenta; eliminamos entonces a los menores de 35 (drop if $\mathrm{v} 05 \mathrm{a}<35)$ y luego a los mayores de 50 (drop if v05a $>50)$.

Para cada encuestado, se tiene una lista de sus ocupaciones (de la primera a la onceava y luego la actual), junto con la edad en la que comenzaron a desempeñarlas. ${ }^{3}$ Pero nosotros necesitamos una estructura de datos distinta, en donde a determinada edad (25 años) queremos conocer la ocupación que se encontraba desempeñando cada persona. 
Cuadro 1. Ejemplos de estructura de datos de trayectorias ocupacionales

\begin{tabular}{|c|c|c|c|c|c|c|c|c|c|c|}
\hline Caso & Oc. 1 & $\begin{array}{c}\text { Edad } \\
\text { oc1. }\end{array}$ & Oc. 2 & $\begin{array}{c}\text { Edad } \\
\text { oc2. }\end{array}$ & Oc. 3 & $\begin{array}{c}\text { Edad } \\
\text { oc3. }\end{array}$ & Oc. 4 & $\begin{array}{c}\text { Edad } \\
\text { oc4. }\end{array}$ & Oc. actual & $\begin{array}{c}\text { Edad } \\
\text { oc Act. }\end{array}$ \\
\hline 1 & Albañil & 16 & Operario & 22 & Chofer & 27 & Taxista & 30 & Comerciante & 35 \\
\hline 2 & $\begin{array}{c}\text { Empleado } \\
\text { administrativo }\end{array}$ & 18 & Contable & 21 & Supervisor & 24 & Gestor & 29 & Contador & 34 \\
\hline 3 & Peón & 15 & Albañil & 19 & Soldador & 22 & Techista & 30 & Soldador & 33 \\
\hline 4 & - & & & - & - & - & - & - & - & Abogado \\
\hline
\end{tabular}

Fuente: elaboración propia.

La variable ocupación a los 25 años puede corresponder, según el encuestado, a cualquiera de sus ocupaciones (de la 1 ra. a la 11va.). Entonces: ¿ cómo hacemos para identificar la ocupación a los 25 años de edad con la estructura de datos que tenemos? En el Cuadro 1, en el caso n. ${ }^{\circ}$ 1, podemos observar que su ocupación a los 25 años fue su segunda ocupación, de operario (que tuvo entre los 22 y 27 años) y que luego comenzó a trabajar de chofer. Pero en el caso n. ${ }^{\circ} 2$, su ocupación a los 25 años es la 3.a (de supervisor). En cambio, si comenzamos por la trayectoria de atrás para adelante (en el cuadro desde la 4ta ocupación, hasta la 1.a) se observa que la ocupación a los 25 años será siempre la anterior a la que primera que se tiene luego de los 25 años.

La forma de generalizar esta idea en una serie de comandos de Stata que permitieran construir la variable fue la siguiente: nos ubicamos en la última ocupación (la última de la trayectoria, sin contar la actual, que será tomada en cuenta luego), si su edad es mayor a 25 entonces la ocupación a los 25 es (por ahora) la anterior a esta. Luego continuamos: nos ubicamos en la anteúltima y si su edad es mayor a 25 entonces la ocupación a los 25 es la anterior a esta (reemplazando si es necesario al valor establecido anteriormente). Realizando este procedimiento "de atrás hacia adelante" de manera sucesiva podemos ir identificando las ocupaciones hasta encontrar exactamente a la ocupación anterior a la primera que supere los 25 años. Con nuestra estructura de datos esta será lógicamente la ocupación que la persona realizaba a los 25 años. Otro tipo de caso posible son aquellos cuya ocupación actual es la misma que tenían a los 25 años; para estos casos sumamos un comando al final que recupera esta información.

Habiendo reconstruido la ocupación a los 25 años de edad (variable llamada o25), y ya teniendo la ocupación actual de la persona (v16), ambas codificadas con el CIUO-08 a 4 dígitos, podemos "reagruparlas" para reducir la cantidad de categorías. Para ello se utilizaron tres comandos (gen, recode y replace). El primer comando (gen ocutr $=\mathrm{v} 16$ ) crea una copia de la variable de ocupación actual sobre la que trabajaremos para llegar a la versión agrupada. Luego recode nos permite agrupar a grandes rasgos cuando los grupos son grandes (por ejemplo, todas las ocupaciones del grupo 1 del CIUO-08, desde la 1000 hasta la 1439 en una sola categoría con recode ocutr $(1000 / 1999=1)$ ). Luego, más específicamente para las categorías que se encuentran compuestas por algunas ocupaciones en particular (como por ejemplo los comerciantes) podemos utilizar el comando replace (replace ocutr $=11$ if v16=5221) que le asigna el valor 11 en la variable de ocupación recodificada (ocutr) si la ocupación actual (v16) es comerciante (5221). Finalmente 
etiquetamos la variable, creamos el sistema de categorías y se lo asignamos a la variable. Posteriormente puede volver a recodificarse esta variable para tener un sistema de categorías aún más reducido ( 5 categorías).

Cuadro 2. Categorías de la variable de trayectoria ocupacional. Frecuencias entre paréntesis

\begin{tabular}{|c|c|c|c|c|c|}
\hline & \multicolumn{5}{|c|}{ Ocupación a los 25 años de edad } \\
\hline Ocupación actual & 1.Medias-Altas & $\begin{array}{l}\text { 2.Medias- } \\
\text { medias }\end{array}$ & $\begin{array}{l}\text { 3.Medias- } \\
\text { Bajas }\end{array}$ & $\begin{array}{l}4 . \mathrm{Ob} \text {. } \\
\text { calificados esp. }\end{array}$ & $\begin{array}{l}\text { 5. Trab. } \\
\text { peones no } \\
\text { cal. }\end{array}$ \\
\hline 1.Medias-Altas & $\begin{array}{l}\text { 1. Reproducción } \\
\text { gerencial- } \\
\text { profesional (36) }\end{array}$ & $\begin{array}{l}\text { 2. Ascenso a } \\
\text { gerencial- } \\
\text { profesional } \\
\text { desde } \\
\text { media-media } \\
\text { (28) }\end{array}$ & \multicolumn{3}{|c|}{$\begin{array}{l}\text { 3. Ascenso a gerencial-profesional desde } \\
\text { media-baja y baja (14) }\end{array}$} \\
\hline 2.Media-Medias & \multicolumn{2}{|c|}{$\begin{array}{l}\text { 4. Reproducción trayectoria } \\
\text { técnico-administrativa ( } 80)\end{array}$} & \multicolumn{3}{|c|}{ 5. Ascenso a técnico-administrativa (34) } \\
\hline 3.Media-Bajas & \multicolumn{3}{|c|}{ 6. Reproducción trayectoria commercial (15) } & \multicolumn{2}{|c|}{$\begin{array}{c}\text { 7. Ascenso leve trayectoria } \\
\text { commercial (18) }\end{array}$} \\
\hline $\begin{array}{l}\text { 4.Ob. calificados } \\
\text { esp. }\end{array}$ & \multicolumn{3}{|c|}{ 8. Descenso trayectoria obrera (25) } & $\begin{array}{c}\text { 9.Reproducción } \\
\text { obrero } \\
\text { consolidado } \\
(100) \\
\end{array}$ & $\begin{array}{l}\text { 10. Ascenso } \\
\text { trayectoria } \\
\text { obrera (12) }\end{array}$ \\
\hline $\begin{array}{l}\text { 5. Trab. peones no } \\
\text { cal. }\end{array}$ & \multicolumn{4}{|c|}{ 11. Descenso a trabajadores no calificados (18) } & $\begin{array}{c}12 . \\
\text { Reproducción } \\
\text { trabajadores } \\
\text { no calificados } \\
(24)\end{array}$ \\
\hline
\end{tabular}

Fuente: elaboración propia.

En la Tabla 2 del Anexo se cruzaron la ocupación a los 25 años y la actual; ella muestra cómo se agruparon las categorías. En el Cuadro 2 se observa cómo se agrupan las celdas de la Tabla 2; cada celda corresponde a una categoría determinada de la variable, y esto es muy simple de traducir en comandos de Stata para generar un indicador de trayectoria ocupacional. Esto se realiza en Stata generando una variable vacía (gen trayectorias=.) y asignando a una categoría de trayectoria cada una de las celdas del cruce entre ocupación actual y ocupación a los 25 años. Por ejemplo, la celda de ocupación actual Media alta y ocupación a los 25 años Media alta corresponde a la primera categoría de la trayectoria (reproducción en la clase media alta) y se le asigna con el siguiente comando: "replace trayectorias $=1$ if ocutra==1 \& o $25 \mathrm{ra}==1$ ". Este procedimiento se realiza para cada una de las celdas del cruce bivariado, asignándoles su categoría de trayectoria correspondiente.

Cada una de las combinaciones antes descritas (Cuadro 2) constituye un tipo de trayectoria desde la ocupación a los 25 años hasta la actual. Mirados en conjunto, sobre los 404 casos el $70 \%$ corresponde a trayectorias de reproducción, el $19.3 \%$ de ascenso y el $10.7 \%$ a casos de descenso. El Cuadro 3 es un ejemplo de cómo se puede usar el indicador; muestra diferencias cuando se tiene en cuenta la edad en que comenzaron a trabajar personas con distintas trayectorias ocupacionales. Aunque el tamaño de la muestra nos lleva a ser prudentes en nuestras afirmaciones, merece destacarse que el inicio en el primer trabajo a los 12 años o antes se observa preferentemente en la clase baja, regularidad que se repite si consideramos 15 o menos años, mientras entre las ocupaciones altas se destaca el inicio a partir de los 19 años (47\%). Como dijimos más arriba, el análisis cualitativo muestra las mismas regularidades. 
Cuadro 3. Edad de la primera ocupación según trayectoria ocupacional

\begin{tabular}{|c|c|c|c|c|c|}
\hline & \multicolumn{5}{|c|}{ Edad de la primera ocupación } \\
\hline Trayectoria ocupacional & $\begin{array}{l}\text { hasta } 12 \\
\text { años de } \\
\text { edad }\end{array}$ & $\begin{array}{l}13,14 \text { y } 15 \\
\text { años de } \\
\text { edad }\end{array}$ & $\begin{array}{c}16,17 \text { y } 18 \\
\text { años de } \\
\text { edad }\end{array}$ & $\begin{array}{l}19 \text { o más } \\
\text { años de } \\
\text { edad }\end{array}$ & Total \\
\hline $\begin{array}{l}\text { 1.Reproducción gerencial- } \\
\text { profesional }\end{array}$ & 1 & 1 & 17 & 17 & 36 \\
\hline $\begin{array}{l}\text { 2. Ascenso a gerencial- } \\
\text { profesional desde media-media }\end{array}$ & 0 & 1 & 16 & 11 & 28 \\
\hline $\begin{array}{c}\text { 3. Ascenso a gerencial- } \\
\text { profesional desde media-baja y } \\
\text { baja }\end{array}$ & 0 & 2 & 5 & 7 & 14 \\
\hline $\begin{array}{l}\text { 4. Reproducción trayectoria } \\
\text { técnico-administrativa }\end{array}$ & 2 & 5 & 40 & 33 & 80 \\
\hline $\begin{array}{l}\text { 5. Ascenso a técnico- } \\
\text { administrativa }\end{array}$ & 1 & 7 & 16 & 10 & 34 \\
\hline $\begin{array}{l}\text { 6. Reproducción trayectoria } \\
\text { comercial }\end{array}$ & 1 & 2 & 5 & 7 & 15 \\
\hline $\begin{array}{l}\text { 7. Ascenso leve trayectoria } \\
\text { comercial }\end{array}$ & 4 & 7 & 7 & 0 & 18 \\
\hline 8. Descenso trayectoria obrera & 2 & 3 & 9 & 11 & 25 \\
\hline $\begin{array}{c}\text { 9.Reproducción obrero } \\
\text { consolidado }\end{array}$ & 12 & 35 & 35 & 18 & 100 \\
\hline 10. Ascenso trayectoria obrera & 2 & 2 & 7 & 1 & 12 \\
\hline $\begin{array}{l}\text { 11. Descenso a trabjadores no } \\
\text { calificados }\end{array}$ & 3 & 6 & 6 & 3 & 18 \\
\hline $\begin{array}{l}\text { 12. Reproducción trabajadores } \\
\text { no calificados }\end{array}$ & 6 & 4 & 7 & 7 & 24 \\
\hline Total & 34 & 75 & 170 & 125 & 404 \\
\hline
\end{tabular}

Fuente: elaboración propia.

\section{REFLEXIONES SOBRE LOS ALCANCES Y LIMITACIONES DEL INDICADOR}

La idea de trayectoria ocupacional induce a pensar en un camino (path) en el tiempo con un inicio y un destino; también es posible pensarla como una serie de eventos o situaciones, en los cuales se desempeñan ocupaciones a lo largo de la vida económicamente activa. A su vez la idea de curso de vida o de historia de vida lleva a pensar siempre en una línea continua de eventos vinculados entre sí a lo largo de múltiples transiciones. El desempeño ocupacional en el curso de vida incluye además de las ocupaciones los periodos de desempleo o licencias. La investigación de trayectorias por lo tanto da lugar a múltiples miradas.

Una de esas miradas es el indicador de corte transversal en el cual se comparan dos momentos en la trayectoria, cuando muy probablemente se han adquirido competencias laborales, ya sea en el ciclo educativo o por aprendizaje en el terreno y la ocupación actual. Los cambios/no cambios entre esas fechas nos permiten delimitar el periodo histórico-económico de los mercados de trabajo, en los cuales se insertan las trayectorias laborales. Este análisis ayuda a comprender algunos aspectos de los modelos de desarrollo económico y el papel de la adquisición de conocimientos y competencias (por ejemplo, adquiridos en el trabajo) en el marco de cómo operan los mercados laborales.

Cuando se trabaja con una muestra que no es muy grande, la decisión de tomar solo dos momentos en la trayectoria nos permite: primero, tratarla como una variable dependiente a ser potencialmente explicada por un conjunto de variables independientes; y segundo, pueden ser analizadas insertándolas en los mercados de trabajo en los cuales muy posiblemente tuvieron lugar. Por ejemplo, establecer los tipos de educación 
y formación asociados a la reproducción o cambio ocupacional. Otro ejemplo: analizar el proceso de feminización de las ocupaciones administrativas (del conjunto de ocupaciones medias-medias) e identificar qué características tienen las mujeres que han ascendido a posiciones gerenciales-profesionales. Otro tema de interés a ser tratado es la descripción de las personas que en su trayectoria han reproducido el desempeño de ocupaciones que los sitúan como obreros calificados/especializados; en qué tipo de empresas y actividades se han desempeñado (información que está disponible en la base de datos).

La manera en que hemos construido el indicador tiene una limitación si deseamos identificar las ocupaciones en las sucesivas entradas y salidas del mercado de trabajo. Este tema es importante en los casos en que operan los mercados laborales de gran inestabilidad; por ejemplo, las tareas de reparaciones, el desempeño en la industria de la construcción y del servicio doméstico.

Como todo indicador de una variable compleja, el nuestro debe ser interpretado con cuidado. Nos brindará mucha información y nos dejará muchas dudas. Una vez que haya sido probado en el campo, los argumentos para confiar en los alcances de su validez provendrán indudablemente de la consistencia teórica de sus resultados y de la compatibilidad con los resultados de otros estudios dentro de la misma temática. Su consistencia y compatibilidad con los estudios históricos constituye también una manera eficiente de confiar en sus resultados.

La fuerza y también las limitaciones de este indicador provienen en buena medida de la construcción de los conjuntos ocupacionales y su asignación a clases o estratos sociales. Al respecto no ha innovado, sigue la tradición de los estudios sobre el tema. Aunque existen algunas pequeñas diferencias en la construcción de las clases y estratos sociales, en nuestro país y a nivel internacional los sistemas clasificatorios basados en el CIUO-OIT constituyen una norma muy generalizada.

\section{ReFERENCIAS}

Billari, F. C. (2001). Sequence analysis in demographic research. Special Issue on Longitudinal Methodology, Canadian Studies in Population, 28(2), 439-458. Recuperado de: https://ejournals.library.ualberta.ca/index.php/csp/arti cle/download/15880/12685

Brzinsky-Fay C. \& Kohler, U. (2010). New Developments in Sequence Analysis. Sociological Methods \& Research, 38(3), 359-364. Recuperado de: https://n9.cl/ez8s

Busso, M., Bidart, C., Longo, M.E. y Perez, P. (2011).La estabilidady la inestabilidad en los procesos de inserción laboral dejóvenes en Argentina y en Francia. Ponencia presentada en PROYECTO ECOS: Informalización, precariedad: el trabajo en la globalización, Montevideo. Recuperado de: https://ri.conicet.gov.ar/handle/11336/4992

Cano, A. J. (2018). Las trayectorias educativas de hombres y mujeres jóvenes. Una aproximación desde el análisis de secuencias. Papers. Revista de Sociologia, 103(1), 5-28. Recuperado de: https://papers.uab.cat/article/downloa $\mathrm{d} / 2290 / 2168$

Danani, C., y Lindenboim, J. (2003). Trabajo, política y políticas sociales en los 90: ¿hay algo de particular en el caso argentino? En C. Dananiy J. Lindemboin (comps.), Entre el trabajo y la política. Las reformas de las políticas sociales argentinas en perspectiva comparada. Buenos Aires: Biblos. Recuperado de http://academia.edu/download/60051247/18..Entre_el_trab_y_la_politica_-_Cap_final_-C_Danani_y_J L_2003pdf20190718-78450-piphir.pdf

Elder, G., Johnson, M. \& Crosnoe, R. (2006). The emergent and development of life course theory. En J. Mortimer \& M. Shanahan, Handbook of the life course (pp. 3-19). N.Y.: Springer.

Gabadinho, A., Ritschard, G., Müller, N. S. \& Studer, M. (2011). Analyzing and visualizing state sequences in R with TraMineR. Journal of Statistical Software, 40(4), 1-37. Recuperado de: https://archive-ouverte.unige.ch/unige :16809/ATTACHMENT01

Jacinto, C., y Chitarroni, H. (2010). Precariedades, rotación y movilidades en las trayectorias laborales juveniles. Estudios del Trabajo, 39(40), 5-36. Recuperado de: https://www.academia.edu/download/52723190/Precarie dades_rotacion_y_movilidades_en_las_trayectorias_laborales_juveniles.pdf 
Jiménez, J. (2016). Más allá de la Observación Dual. Situación y trayectorias de los trabajadores por cuenta propia en Chile. 9. o Congreso de Sociología en Chile, Talca 11-14 Octubre de 2016. GT 20 Procesos, sujetos y condiciones del trabajo en Chile. Recuperado de: http://www.academia.edu/download/49639391/GT_20.pdf

Karlson, K. B., \& Birkelund, J. F. (2019). Education as a mediator of the association between origins and destinations: The role of early skills. Research in Social Stratification and Mobility, 64.

Lindenboim, J. (2001). Mercados de trabajo urbanos en Argentina de los 90. Crisis y metamorfosis del mercado de trabajo, 13. Recuperado de: http://157.92.136.59/download/docin/docin_ceped_c_004.pdf\#page=5

Lindenboim, J., y Salvia, A. (2017). Hora de balance: proceso de acumulación, mercado de trabajo y bienestar: Argentina, 2002-2014. Buenos Aires: Eudeba.

Muñiz Terra, L. (2012). Carreras y trayectorias laborales: una revisión crítica de las principales aproximaciones teóricometodológicas para su abordaje. Revista latinoamericana de metodología de las Ciencias Sociales, 2(1), 36-65. Recuperado de http://www.memoria.fahce.unlp.edu.ar/art_revistas/pr.5218/pr.5218.pdf

Rohwer, G. \& Trappe, H. (1999). Possibilities and difficulties in life course description, en W. Voges (Ed.). Dynamic approaches to comparative social research. Recent developments and applications, pp. 146-167. Aldershot: Avebury Publishers.

Sautu, R. (2011). El análisis de las clases sociales: teorías y metodologías. Ciudad Autónoma de Buenos Aires: Ediciones Luxemburg.

Sautu, R. (2016). Economía, clases sociales y estilos de vida. Ciudad Autónoma de Buenos Aires: Lumiere.

Sautu, R. (2020). Trayectorias ocupacionales en su contexto histórico. Varones adultos jóvenes residentes en el Area Metropolitana de Buenos Aires. En evaluación.

Sautu, R., Boniolo, P. Dalle, P. y Elbert, R. (2020). El análisis de clases sociales. Pensando la movilidad, la residencia, los lazos sociales, la identidad y la agencia. Ciudad Autónoma de Buenos Aires: Universidad de Buenos Aires. Instituto de Investigaciones Gino Germani - UBA.

Solís, P., y Billari, F. C. (2003). Vidas laborales entre la continuidad y el cambio social: trayectorias ocupacionales masculinas en Monterrey, México. Estudios Demográficos y Urbanos, 18-3(54), 559-595.

Struffolino, E. (2019). Navigating the early career: The social stratification of young workers' employment trajectories in Italy. Research in Social Stratification and Mobility, 63, 10. 


\section{Anexo}

Tabla 1. Código de ocupaciones CIUO-08 agrupadas

\begin{tabular}{|c|c|c|}
\hline Códigos CIUO-08 & & Categoría \\
\hline & OCUPACIONES MEDIAS-ALTAS & \\
\hline 1000 & Directores y gerentes públicos y privados & 1 \\
\hline 2100 & Profesionales de la ciencia y de la ingeniería & 2 \\
\hline 2200 & Profesionales de la salud & 3 \\
\hline 2400 más 2500 más 2600 & Prof. derecho, administración & 5 \\
\hline & OCUPACIONES MEDIA-MEDIA & \\
\hline 2300 & & 4 \\
\hline 3000 & & 6 \\
\hline 4000 (exœepto los siguientes) & Administrativos & 7 \\
\hline 4110 & Oficinistas generales & 8 \\
\hline $4310(4311,4312 y 4313)$ & Auxiliares contables y financieros & 9 \\
\hline \multirow[t]{2}{*}{0} & Ocupaciones militares & 23 \\
\hline & OCUPACIONES MEDIA-BAJA & \\
\hline 5221 & Comerciantes de tiendas & 11 \\
\hline \multirow[t]{2}{*}{5223} & Asistentes de venta de tiendas y almacenes & 12 \\
\hline & TRABAJADORES-OBREROS CALIFICADOS-ESPECIALIZADOS & \\
\hline 5000 (excepto 5221 y 5223) & Trabajadores de los servicios personales & 10 \\
\hline 6000 & Agricultores y trabajadores calificados agropecuarios, etc. & 13 \\
\hline 7000 (exœepto los siguientes) & & 14 \\
\hline 7112 & & 15 \\
\hline 7210 (7211 hasta 7215) & Moldeadores, soldadores, chapistas, caldereros, etc. & 16 \\
\hline 7510 (7511 hasta 7516) & Oficiales y operarios en alimentos y bebidas & 17 \\
\hline \multirow[t]{2}{*}{8000} & Operadores de instalaciones y maquinas & 18 \\
\hline & TRABAJADORES-PEONES NO CALIFICADOS & \\
\hline 9000 (exœepto los siguientes) & & 19 \\
\hline 9111 & Limpiadores y asistentes domésticos & 20 \\
\hline 9112 & Limpiadores y asistentes de oficinas, de hoteles etc. & 21 \\
\hline 9621 & Mensajeros, mandaderos, maleteros, repartidores & 22 \\
\hline
\end{tabular}

NOTA: Las ocupaciones que tienen los mayores vínculos con otras ocupaciones han sido identificadas separadamente (Sautu, Carrascosa, Paredes, 2020). 


\begin{tabular}{|c|c|c|c|c|c|c|c|c|c|c|c|c|c|c|c|c|c|c|c|c|c|c|c|c|c|c|c|c|c|}
\hline \multirow[b]{2}{*}{ Ocupación actual } & \multicolumn{29}{|c|}{$\begin{array}{l}\text { TABLA 2. TABULADO DE LA DISTRIBUCION DE OCUPACIONES ACTUALES CON OCUPACIONES ALOS } 25 \text { AÑOS DE EDAD } \\
\text { Ocupación a los } 25 \text { años }\end{array}$} \\
\hline & 1. & 1 & 2 & 3 & 5 & \multirow[t]{2}{*}{2} & 4 & 6 & 7 & 8 & 9 & 23 & \multirow[t]{2}{*}{3} & 11 & 12 & \multirow[t]{2}{*}{4} & 10 & 13 & 14 & 15 & 16 & 17 & 18 & \multirow[t]{2}{*}{5} & \multirow[t]{2}{*}{19} & \multirow[t]{2}{*}{20} & \multirow[t]{2}{*}{21} & \multirow[t]{2}{*}{22} & \multirow[t]{2}{*}{$\mathrm{T}$} \\
\hline \multicolumn{21}{|l|}{ 1.Medias altas } & & & & & & & & & \\
\hline 1. Diry gerentes & & 6 & 0 & 0 & 2 & & 0 & 5 & 0 & 2 & 1 & 0 & & 2 & 1 & & 1 & 0 & 0 & 0 & 0 & 0 & 0 & & 1 & 0 & 0 & 0 & 21 \\
\hline $\begin{array}{l}\text { 2.Prof ciencia e } \\
\text { ingeniería }\end{array}$ & & 0 & 3 & 0 & 0 & & 1 & 1 & 0 & 0 & 0 & 0 & & 0 & 0 & & 1 & 0 & 0 & 0 & 0 & 0 & 1 & & 0 & 0 & 0 & 0 & 7 \\
\hline 3.Prof de la salud & & 0 & 0 & 3 & 0 & & 1 & 0 & 0 & 1 & 0 & 0 & & 0 & 0 & & 0 & 0 & 0 & 0 & 0 & 0 & 0 & & 0 & 0 & 0 & 0 & 5 \\
\hline 5.Prof. 2400 a 2600 & & 1 & 0 & 0 & 21 & & 1 & 6 & 4 & 5 & 0 & 0 & & 1 & 2 & & 1 & 0 & 1 & 0 & 0 & 1 & 0 & & 0 & 0 & 0 & 1 & 45 \\
\hline \multicolumn{30}{|l|}{ 2. Medias-Medias } \\
\hline 4.Prof de la enseñanza & & 1 & 0 & 0 & 0 & & 18 & 2 & 1 & 3 & 0 & 0 & & 0 & 2 & & 5 & 0 & 1 & 0 & 0 & 0 & 0 & & 0 & 1 & 0 & 0 & 34 \\
\hline 6. Técnicos y prof nivel & & 0 & 0 & 0 & 2 & & 0 & 12 & 0 & 2 & 1 & 0 & & 1 & 2 & & 2 & 0 & 3 & 0 & 0 & 1 & 2 & & 1 & 1 & 0 & 0 & 30 \\
\hline 7.Administativos & & 0 & 0 & 0 & 0 & & 1 & 2 & 7 & 5 & 0 & 0 & & 0 & 2 & & 3 & 0 & 0 & 0 & 0 & 0 & 2 & & 0 & 1 & 0 & 1 & 24 \\
\hline 8. Oficinistas & & 0 & 0 & 0 & 0 & & 1 & 1 & 1 & 9 & 1 & 0 & & 0 & 0 & & 1 & 0 & 0 & 0 & 0 & 0 & 0 & & 0 & 0 & 0 & 0 & 14 \\
\hline 9.Aux contables $y$ fina & & 0 & 0 & 0 & 0 & & 0 & 0 & 0 & 0 & 9 & 0 & & 0 & 1 & & 1 & 0 & 0 & 0 & 0 & 0 & 0 & & 0 & 0 & 0 & 0 & 11 \\
\hline 23. Ocup militares & & 0 & 0 & 0 & 0 & & 0 & 0 & 0 & 0 & 0 & 1 & & 0 & 0 & & 0 & 0 & 0 & 0 & 0 & 0 & 0 & & 0 & 0 & 0 & 0 & 1 \\
\hline \multicolumn{30}{|l|}{ 3. Medias-bajas } \\
\hline 11.Comeriantes & & 0 & 0 & 0 & 0 & & 0 & 1 & 1 & 2 & 0 & 0 & & 3 & 3 & & 2 & 0 & 1 & 1 & 0 & 0 & 3 & & 2 & 3 & 0 & 0 & 22 \\
\hline 12.Vendedores & & 0 & 0 & 0 & 0 & & 0 & 0 & 0 & 0 & 0 & 0 & & 0 & 5 & & 2 & 0 & 0 & 0 & 0 & 0 & 1 & & 1 & 2 & 0 & 0 & 11 \\
\hline \multicolumn{13}{|c|}{ 4.Trab. Obreros calificados } & & & & & & & & & & & & & & & & & \\
\hline 10.Trab. de servicios & & 0 & 0 & 0 & 0 & & 0 & 1 & 3 & 2 & 0 & 0 & & 3 & 6 & & 23 & 0 & 2 & 1 & 0 & 0 & 5 & & 2 & 3 & 0 & 0 & 51 \\
\hline 13.Agricultores y trab. & & 0 & 0 & 0 & 0 & & 0 & 0 & 0 & 0 & 0 & 0 & & 0 & 2 & & 0 & 1 & 0 & 1 & 1 & 0 & 0 & & 0 & 0 & 0 & 0 & 5 \\
\hline 14. Oficiales, operarios & & 0 & 0 & 0 & 0 & & 0 & 0 & 0 & 0 & 0 & 0 & & 1 & 1 & & 2 & 1 & 25 & 1 & 0 & 0 & 5 & & 0 & 1 & 0 & 0 & 37 \\
\hline 15.Albañiles & & 0 & 0 & 0 & 0 & & 0 & 1 & 0 & 0 & 0 & 0 & & 0 & 0 & & 0 & 0 & 0 & 7 & 0 & 0 & 1 & & 2 & 0 & 0 & 0 & 11 \\
\hline $\begin{array}{l}\text { 16.Soldadores, } \\
\text { chapistas }\end{array}$ & & 0 & 0 & 0 & 0 & & 0 & 0 & 0 & 0 & 0 & 0 & & 0 & 0 & & 0 & 0 & 0 & 0 & 1 & 0 & 0 & & 0 & 0 & 0 & 0 & 1 \\
\hline 17. Of. y. Op. en alimento & & 0 & 0 & 0 & 0 & & 0 & 0 & 0 & 0 & 0 & 0 & & 0 & 0 & & 0 & 0 & 1 & 0 & 0 & 2 & 0 & & 0 & 0 & 0 & 0 & 3 \\
\hline 18. Operadores de inst. & & 0 & 0 & 0 & 0 & & 0 & 0 & 0 & 0 & 1 & 0 & & 1 & 3 & & 3 & 0 & 3 & 0 & 0 & 0 & 14 & & 1 & 0 & 0 & 3 & 29 \\
\hline \multicolumn{30}{|c|}{ 5. Trab peones no calificados } \\
\hline $\begin{array}{l}\text { 19. Ocupaciones } \\
\text { elemental }\end{array}$ & & 0 & 0 & 0 & 0 & & 0 & 0 & 0 & 0 & 0 & 0 & & 0 & 0 & & 1 & 0 & 0 & 0 & 0 & 1 & 0 & & 3 & 0 & 1 & 0 & 6 \\
\hline $\begin{array}{l}\text { 20.Limpiadores y } \\
\text { asisten }\end{array}$ & & 0 & 0 & 0 & 0 & & 0 & 1 & 0 & 0 & 0 & 0 & & 1 & 3 & & 3 & 0 & 0 & 0 & 2 & 0 & 3 & & 0 & 16 & 1 & 0 & 30 \\
\hline 21.Limpieadores de ofici & & 0 & 0 & 0 & 0 & & 0 & 0 & 0 & 0 & 0 & 0 & & 0 & 0 & & 0 & 0 & 1 & 0 & 0 & 1 & 0 & & 0 & 2 & 1 & 0 & 5 \\
\hline 22.Mensajeros & & 0 & 0 & 0 & 0 & & 0 & 0 & 0 & 0 & 0 & 0 & & 0 & 0 & & 1 & 0 & 0 & 0 & 0 & 0 & 0 & & 0 & 0 & 0 & 0 & 1 \\
\hline Total & & 8 & 3 & 3 & 25 & & 23 & 33 & 17 & 31 & 13 & 1 & & 13 & 33 & & 52 & 2 & 38 & 11 & 4 & 6 & 37 & & 13 & 30 & 3 & 5 & 404 \\
\hline
\end{tabular}

\section{Notas}

1 La matriz de datos incluye la reconstrucción retrospectiva de sus trayectorias ocupacionales, lo cual permite su ubicación en el contexto económico-histórico de los mercados laborales en el AMBA durante el transcurso de la vida económicamente activa de las personas encuestadas. El objetivo de esa investigación, denominada Pi Clases, es el análisis de las clases sociales, los procesos de movilidad inter e intrageneracional, los efectos territoriales en los procesos de desigualdad y de clase social, los lazos sociales, el papel de la estructura y la agencia, y la identidad de clase (Sautu, Boniolo, Dalle y Elbert, 2020).

2 En el estudio de las trayectorias completas, una opción es usar el paquete de R TraMineR para minar, analizar y visualizar en un gráfico las secuencias de datos categóricos (Gabadinho, Ritschard, Müller, \& Studer, 2011). Este paquete permite organizar y representar gráficamente las distintas trayectorias laborales de una población; es posible también cuando el tamaño de la muestra lo permite, tener en cuenta distintas características como su clase de destino y su cohorte de nacimiento, etc. El paquete Traminer R se complementa con el análisis de cluster (Brzinsky-Fay \& Kohler, 2010) aplicado al total de las trayectorias incluidas en la muestra analizada. Ver por ejemplo Cano (2018); Jiménez (2016), Solís y Billari (2003).

3 No se tiene información sobre momentos en que la persona no trabajó (por ejemplo, por situaciones de desempleo). 